\title{
Development of a hybrid PSO-ANN model for estimating glucose and xylose yields for microwave-assisted pretreatment and the enzymatic hydrolysis of lignocellulosic biomass
}

\begin{abstract}
In this paper, two artificial intelligent systems, the artificial neural network (ANN) and particle swarm optimization (PSO), were combined to form a hybrid PSO-ANN model that was used to improve estimates of glucose and xylose yields from the microwave-acid pretreatment and enzymatic hydrolysis of lignocellulosic biomass based on pretreatment parameters. ANN is a powerful tool capable of determining the relationship between the desired input and output data while PSO was used as a robust population-based search algorithm to optimize the performance of the ANN model. Specifically, it was used to determine the optimum number of neurons in the hidden layer and the best value of the learning rate of the ANN model. The optimization method includes minimizing the fitness function mean absolute error that was found to be 0.0176. The PSO algorithm suggested an optimum number of neurons in the hidden layer as 15 and a learning rate of 0.761 these consequently used to construct the ANN model. After constructing the hybrid PSO-ANN model, the performance of the intelligent system was examined by determining the regression coefficient $\left(R^{2}\right)$ for estimating the experimental values of glucose and xylose and compared to the results from a response surface methodology (RSM) model. The results of $R^{2}$ of the hybrid PSO-ANN model for glucose and xylose were 0.9939 and 0.9479 , respectively, while the RSM model results for the same sugars were 0.8901 and 0.8439 . This analysis reveals that the hybrid PSO-ANN model offers a higher degree of accuracy in comparison with the more commonly used RSM model.
\end{abstract}

Keyword: Hybrid PSO-ANN model; ANN model; Artificial neural network; Glucose; Xylose yields; Microwave-assisted pretreatment; Enzymatic hydrolysis; Lignocellulosic biomass 